Article:

\title{
Poly(ionic liquid) thermo-responsive hydrogel microfluidic actuators
}

Alexandru Tudor, Janire Saez, Larisa Florea, Fernando BenitoLopez, Dermot Diamond

Sensors and Actuators B: Chemical 247: 749-755 (2017)

This work is made available online in accordance with publisher policies. To see the final version of this work please visit the publisher's website. Access to the published online version may require a subscription.

\section{Link to publisher's version:}

https://doi.org/10.1016/j.snb.2017.03.045

Copyright statement: (c) 2017 Elsevier Ltd. Full-text reproduced in accordance with the publisher's self-archiving policy.

This manuscript version is made available under the CC-BY-NC-ND 4.0 license http://creativecommons.org/licenses/by-nc-nd/4.0

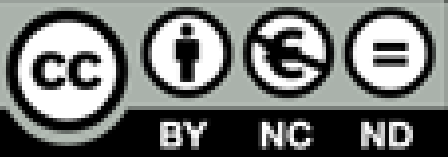




\title{
Poly(ionic liquid) thermo-responsive hydrogel microfluidic valves
}

By Alexandru Tudor ${ }^{\dagger}$, Janire Saez ${ }^{\dagger b}$, Larisa Florea ${ }^{a^{*}}$, Fernando Benito-Lopez $z^{b^{*}}$ and Dermot Diamond $^{a}$

${ }^{a}$ Insight Centre for Data Analytics, National Centre for Sensor Research, Dublin City University, Dublin 9, Ireland.

${ }^{b}$ Analytical Microsystems \& Materials for Lab-on-a-Chip (AMMa-LOAC) Group, Microfluidics UPV/EHU Cluster, Analytical Chemistry Department, University of the Basque Country UPV/EHU, Vitoria-Gasteiz, Spain.

$\uparrow$ Authors contributed equally to this work

*Corresponding author E-mail: larisa.florea@dcu.ie, fernando.benito@ehu.eus

\begin{abstract}
In this study we report the synthesis, characterisation and performance of thermoresponsive crosslinked tributylhexyl phosphonium sulfopropylacrylate (PSPA) poly(ionic liquid) (PILc) hydrogels as temperature controlled valves in microfluidic devices. The hydrogel size is modulated by localised changes in its temperature due to the lower critical solution temperature (LCST) behaviour exhibited by PSPA.
\end{abstract}

Keywords: (Microfluidics, Actuators, Smart Materials, Temperature Responsive Materials, Ionic Liquids, Poly(ionic liquid)s) 


\section{Introduction}

Microfluidics is an interdisciplinary science that focuses on producing devices capable of manipulating small volumes of fluids, ranging from picoliters to microliters ${ }^{1,2}$. The advantages of microfluidics come either directly from the reduction in size compared to conventional laboratory fluidics or as a result of the ability to integrate sequential processes at the micro-scale. The former results in reduced reagent consumption, low waste production, reduced measurement times and improved sensitivity, while the latter offers the benefits of sampling, pre-processing and sample analysis "on-chip", thereby reducing process time, risk of contamination, and human error ${ }^{1-10}$. Despite many years of research, functioning devices incorporating microfluidics still typically require highly specialised and bulky macroscopic equipment such as pumps, valves and detectors to be located off-chip. Therefore, one of the main challenges in microfluidics is downscaling conventional pumps and valves so they can be fully integrated within the microfluidic device. In this context, one of the approaches that are being explored is the use of soft materials for flow control ${ }^{11-17}$. In recent years, effective fluidic control systems based on soft, polymer gel-type materials fully integrated within the microfluidic device have been demonstrated by several groups. These novel devices are now becoming more reminiscent of biological units than conventional silicon micromachined components. Examples include the microfluidic integration of a variety of stimuli-responsive materials that respond to changes in their external environment, such as temperature, light, $\mathrm{pH}$, or variations in electrical potential and pressure ${ }^{18-32}$. Among them, one of the most studied stimuli-responsive hydrogels is poly(N-isopropyl acrylamide) (pNiPAAm), due to its lower critical solution temperature (LCST) behaviour in the presence of an aqueous medium $^{20,33-35}$. LCST behaviour arises from changes in the hydration forces between the polymer chains and the aqueous medium, and the attractive forces between the polymer chains themselves. If the temperature of the hydration medium is below the LCST, then the 
polymer chains are hydrated and swollen by the aqueous medium. When the temperature rises above the LCST, the polymer chains expel the water molecules and attract each other, which leads to the polymer collapsing from solution and contraction of the polymer ${ }^{20,33-35}$. In the case of a linear polymer, the presence of a LCST leads to the appearance of a polymer precipitate, while in the case of a crosslinked polymeric hydrogel, it causes volume contraction $^{31,34}$. This property has made pNiPAAm hydrogels interesting candidates for the realisation of thermo-responsive microfluidic valves and for thermo-, photo- $^{36}$, magnetoresponsive valving units when ionic liquids ${ }^{37}$, photochromic molecules ${ }^{14,38}$, or magnetic nanoparticles ${ }^{39,40}$, respectively, are included in the polymer matrix.

Similar LCST behaviour has been reported recently in a relatively new class of materials, namely poly(ionic liquid)s (PILs) ${ }^{22,29-31,41-43}$. These materials are polymers formed through the polymerisation of ionic liquid (IL) monomers which feature a polymerisable group in the anion, the cation or both. Although generally the focus remains on linear PILs, there are several reports dealing with crosslinked PIL networks, that are explored either as ion conductors ${ }^{44,45}$, templates for multilamellar structured nanoparticles ${ }^{46}$ or as actuators ${ }^{31,47}$.

The aim of this paper is the integration, for the first time, of the phosphonium-based crosslinked PIL tributylhexylphosphonium sulfopropylacrylate (PSPA), in a microfluidic structure, to provide thermo-actuated valve functionality.

\section{Materials and Methods}

\subsection{Materials and reagents}

Potassium 3-sulfopropyl acrylate (KSPA), 2-hydroxy-2-methylpropiophenone 97\% (HMPP), phenyl-bis(2,4,6-trimethylbenzoyl) phosphine oxide 97\% (PBPO), polypropylene glycol diacrylate $\left(\mathrm{M}_{\mathrm{w}} \sim 800,100 \mathrm{ppm}\right.$ MEHQ and $100 \mathrm{ppm}$ BHT as inhibitors) (PPG800) and HPLC grade acetonitrile (ACN) were bought from Sigma Aldrich ${ }^{\circledR}$ (Arklow, Ireland) and 
used as received. Tributylhexyl phosphonium chloride was kindly donated by $\mathrm{Cytec}^{\circledR}$ Industries (Niagara Falls, Canada). Deionized water (DI water), with a resistivity of 18.2 $\mathrm{M} \Omega \cdot \mathrm{cm}^{-1}$, was made using a Milli-Q Water Purification System (Merck Millipore, Darmstadt, Germany).

\subsection{Synthesis of the tributylhexyl sulfopropyl acrylate (PSPA) ionic liquid monomer} For PSPA synthesis, $15 \mathrm{~g}$ of tributylhexyl chloride $(\mathrm{PCl})$ and $16.02 \mathrm{~g}$ of KSPA were dissolved in $25 \mathrm{~mL}$ DI water. The amount of KSPA used represents a 50\% molar excess compared to the stoichiometric value needed for this reaction. This was done to ensure that the reaction equilibrium is shifted towards the formation of PSPA. The reaction was maintained at room temperature and atmospheric pressure overnight (Scheme 1). Purification was done by extracting the PSPA from the aqueous solution with dichloromethane. This process was repeated three times. The resulting mixture was dried over anhydrous magnesium sulphate and gravity filtered. Following this, the extraction solvent was removed by rotary evaporation and the resulting viscous mixture was dried overnight using a high vacuum pump. The obtained product was a clear, colourless viscous liquid. The yield of the synthesis was $\sim 85 \%$. The purity of the product was analyzed using ${ }^{1} \mathrm{H}-\mathrm{NMR}, \delta \mathrm{H}$ (Bruker Advance Ultrashield, $400 \mathrm{MHz}$, deuterated chloroform): 0.86-1 (m, 12H, $\left.\mathrm{CH}_{3}\right), 1.28-1.33$ (m, $\left.4 \mathrm{H}, \mathrm{CH}_{2}\right), 1.51-1.53\left(\mathrm{~m}, 16 \mathrm{H}, \mathrm{CH}_{2}\right), 2.18-2.37\left(\mathrm{~m}, 10 \mathrm{H}, \mathrm{CH}_{2}\right), 2.88-2.92\left(\mathrm{~m}, 2 \mathrm{H}, \mathrm{CH}_{2}\right)$, 4.25-4.29 (t, J=6.46 Hz, 2H, CH $), 5.77-5.80(\mathrm{dd}, 1 \mathrm{H}, \mathrm{J}=1.51,10.42 \mathrm{~Hz}, \mathrm{CH}), 6.04-6.11(\mathrm{~m}$, $1 \mathrm{H}, \mathrm{J}=10.44,17.33 \mathrm{~Hz}, \mathrm{CH}), 6.34-6.39$ (dd, $1 \mathrm{H}, \mathrm{J}=1.51,17.38 \mathrm{~Hz}, \mathrm{CH}) \mathrm{ppm}$. 


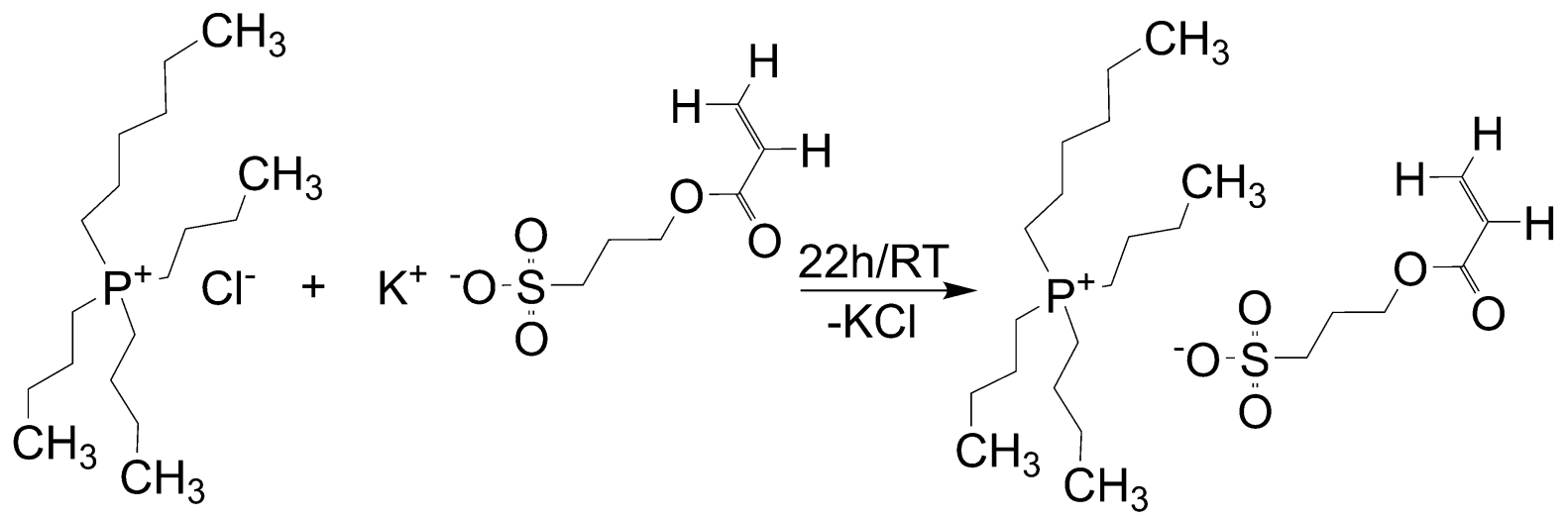

Scheme 1. Reaction scheme for PSPA synthesis.

\subsection{Crosslinked PSPA hydrogel disk polymerization}

The crosslinked PILc hydrogel was synthesized by dissolving $0.1935 \mathrm{~g}$ (400 $\mu \mathrm{mols}$ )

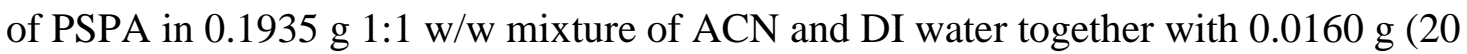
$\mu \mathrm{mols})$ of PPG800 as the crosslinker and $0.0034 \mathrm{~g}$ ( $8 \mu \mathrm{mols})$ of PBPO as the photopolymerization initiator. The resulting mixture was mechanically stirred until all the components were completely dissolved. Following this, the monomer solution was pipetted in poly(dimethyl siloxane) moulds $3 \mathrm{~mm}$ wide and $1 \mathrm{~mm}$ deep (Fig. 1). To polymerize the monomer mixture, a Dolan-Jenner LMI-6000 Fiber-Lite (Boxborough, MA, USA) whitelight was used at an illumination level of $\sim 200 \mathrm{kLux}$ for 30 minutes. The resulting PILc disks were swollen in DI water overnight (Fig. 1). Based on previously done studies ${ }^{48}$, a polymerization time of 30 minutes ensures that the monomer mixture is fully polymerized. 

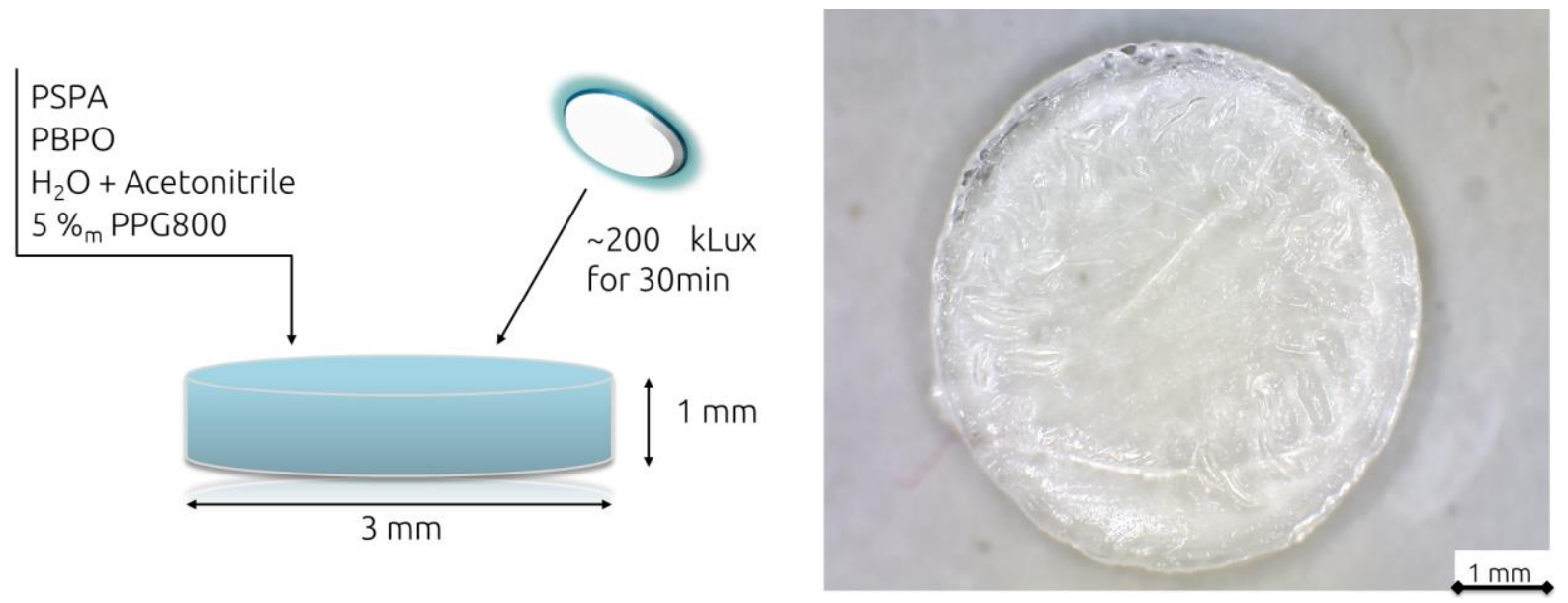

Fig. 1. Schematic illustration of PSPA hydrogel disk polymerization protocol (left) and photo of the PSPA hydrogel disk (right).

\subsection{Characterization of the temperature-induced shrinking/reswelling of the hydrogel} disks

The resulting hydrogel disks, as seen in Fig. 1, were transferred to an Anton Paar MCR301 rheometer fitted with a Peltier temperature control holder with an aluminium plate. The hydrogel disks were covered with DI water and the holder was covered with a $5 \mathrm{~mm}$ glass plate, to ensure that the water will not evaporate when the temperature was raised. Two different experiments were performed: the first experiment focused on the study of the shrinking and swelling kinetics of the hydrogel disks, while the second experiment focused on the repeatability and the reproducibility of the shrinking and reswelling effect. The first experiment was carried out by heating the hydrogels from $20{ }^{\circ} \mathrm{C}$ to $70{ }^{\circ} \mathrm{C}$ in $5{ }^{\circ} \mathrm{C}$ steps to analyze the shrinking effect, followed by cooling the gels at the same rate back to $20{ }^{\circ} \mathrm{C}$ to analyze the reswelling effect. The hydrogel disks were kept for 8 minutes at each step to ensure that the area of the hydrogel has reached a steady state. At each step an image was taken using an Aigo GE-5 digital microscope having a 60x magnification objective. The hydrogels' \%shrinking in area was studied using the ImageJ software. Each image was analyzed using the following formula: 


$$
\% \text { shrinking }=100-\left(A_{f} / A_{i} \cdot 100\right)
$$

Where $A_{f}$ is the size of the shrunken hydrogel disk at a set temperature and $A_{i}$ is the initial size of the swollen hydrogel disk.

To further illustrate this behaviour, a time-lapse video of the gel shrinking and reswelling was made by using images taken every 20 seconds for a total of 60 minutes for each shrinking and reswelling cycle (Video S1: Temperature-induced shrinking and reswelling of PILc hydrogels).

The second experiment was done by alternatively shrinking the hydrogel disks by applying a temperature of $50{ }^{\circ} \mathrm{C}$ and reswelling them by applying a temperature of $20{ }^{\circ} \mathrm{C}$. This alternation was repeated six times to ensure that the temperature-induced shrinking behaviour is repeatable. The same formula (Eq. 1) was used in this case to calculate \%shrinking in area when the hydrogels were heated to $50{ }^{\circ} \mathrm{C}$.

\subsection{Microfluidic chip fabrication}

The microfluidic chip was fabricated from poly(methyl methacrylate) (PMMA) sheets with a height of $250 \mu \mathrm{m}$ and pressure sensitive adhesive (PSA) sheets with a thickness of 60 $\mu \mathrm{m}$. The bottom parts of the chip, the well layer in which the hydrogel was polymerized and the top part of the chip were cut using an Epilog Zing $1630 \mathrm{~W} \mathrm{CO}_{2}$ laser. These three PMMA layers were bound using two PSA layers, the first of which was used to connect the bottom part of the chip to the polymer well layer of the chip, while the second PSA layer had the microfluidic channel cut into it and was used to connect the top part of the chip to the polymer well layer of the chip (Fig. 2). The PSA layers were cut using a Graphtec Craft Robo-Pro cutting plotter. The total thickness of the chip upon assembly (Fig. 3) was $1 \mathrm{~mm}$. 
This dimension was used to ensure the chip will fit in the microfluidic chip holder which will be used to actuate the thermo-responsive hydrogel valve.

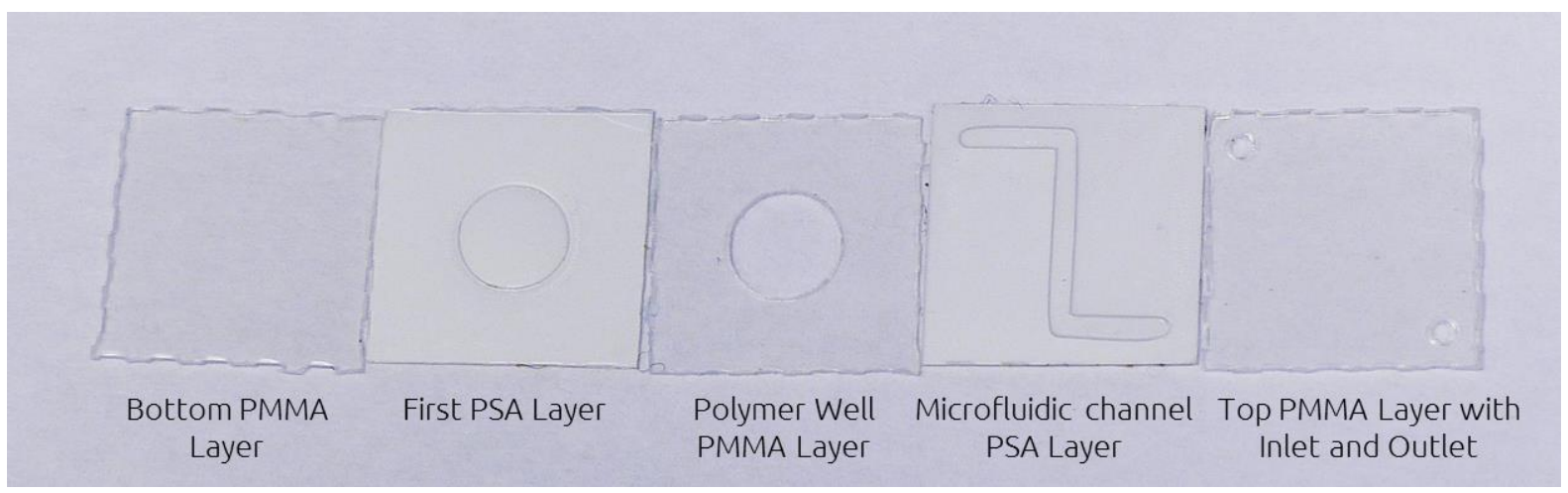

Fig. 2. PMMA and PSA layers of the microfluidic chip before assembly.

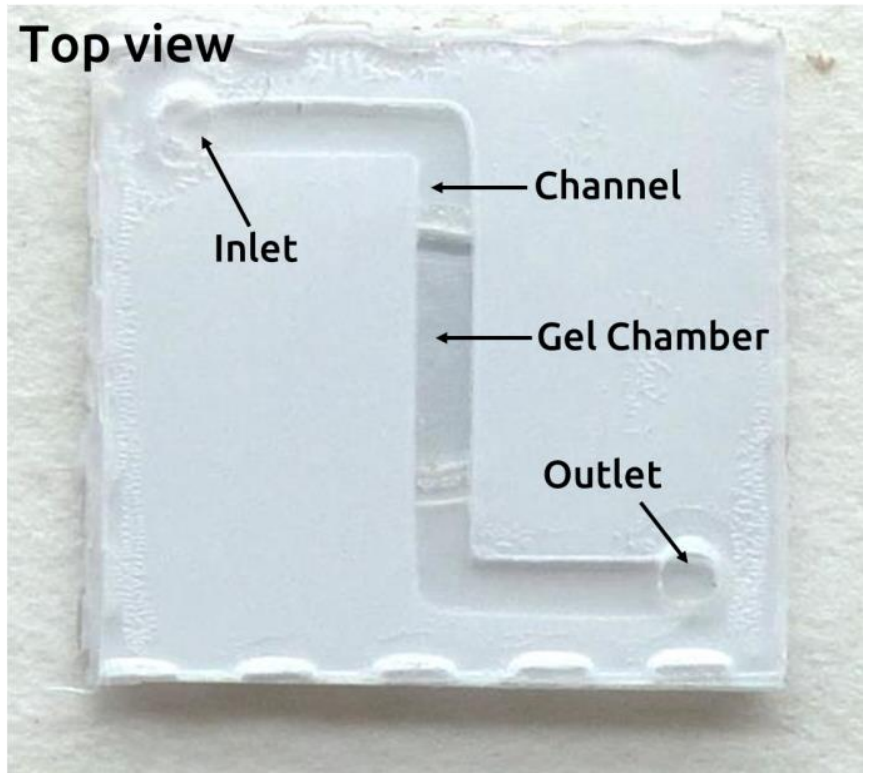

Fig. 3. The assembled microfluidic chip containing the thermo-responsive hydrogel valve.

\subsection{Thermo-responsive hydrogel valve characterization}

The hydrogel valves were fabricated by photo-polymerizing $3 \mu \mathrm{L}$ of monomer mixture for 20 min using a 365nm UV lamp. In this case, the PBPO initiator was substituted, while respecting the same mol ratio, with HMPP to allow UV photopolymerization. Based on 
a previously published study ${ }^{49}$, the polymerization time used ensures the full monomer conversion. The characterization of the valve's thermo-response was done by inserting the microfluidic chip into a microfluidic holder fitted with a heating element. This heating element sits at the bottom of the microfluidic chip and is capable of homogenously increasing the temperature at a rate of $7.4{ }^{\circ} \mathrm{C} \cdot \mathrm{s}^{-1}$ and decreasing the temperature at a rate of $2.4{ }^{\circ} \mathrm{C} \cdot \mathrm{s}^{-1}$. To induce shrinking in the thermo-responsive PILc hydrogel, a temperature of $50{ }^{\circ} \mathrm{C}$ was chosen, while to reswell the hydrogel a temperature of $20^{\circ} \mathrm{C}$ was chosen. The microfluidic holder also contains the inlet and outlet connections at its sides. After the microfluidic chip was placed in the holder, its inlet was connected to a WPI-Europe SP101IZ syringe pump, while its outlet was connected to a Sensirion CMOSens flow microsensor. The flow rate was set at $500 \mathrm{~nL} \cdot \mathrm{min}^{-1}$. The schematic of the setup can be seen in Scheme 2 .

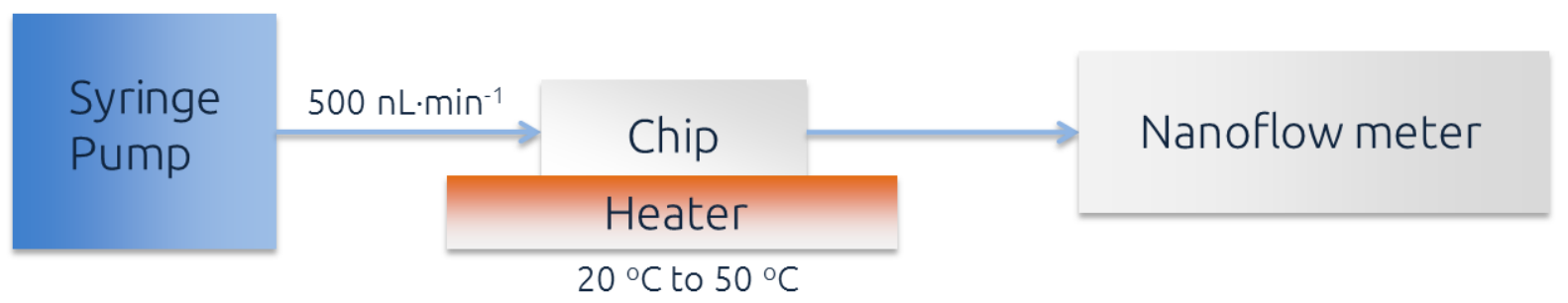

Scheme 2. Setup used for the characterization of DI water flow through the chip.

DI water was chosen as the flow medium to ensure that there are no other effects inhibiting the shrinking behaviour of the PIL hydrogels. The effect of foreign salts dissolved in the hydration medium on the temperature-induced shrinking of both linear and crosslinked PILs were investigated in several other studies ${ }^{18,28,29,48,50}$. For future applications envisioned to work with different kinds of solutions as flow mediums, a configuration in which the hydrogel valve is separated from the flow medium by a flexible membrane can be used, as demonstrated by Tanaka $e t . a l^{51}$ and Beebe $e t . a l^{11}$. In both cases they used a flexible 
membrane to separate an electroactive polymer, and a $\mathrm{pH}$-responsive hydrogel, respectively, from the analyte flow, which ensured that they operated in optimal conditions.

The results on the actuation of the ionic liquid polymer valve followed a sigmoidal fit (Flow rate, $Q$, versus time, $t$ ) (Eq.2). This type of fit is commonly used in chemistry to obtain constant values such as $\mathrm{pK}_{\mathrm{a}}$ (acid dissociation constant) and can be used to determine the rate constant of the ionic liquid polymer valve actuation in this case.

$$
Q=\frac{a}{1+e^{k x+b}}+d
$$

Where " $a$ " is the maximum $Q$ value obtained when valve is fully open, " $b$ " correspond to the interception point value, $k$ give us the value of the rate constant, and " $d$ " accounts for a baseline offset, minimum $Q$.

Using the setup from Scheme 2 the flow rates through the chip were analysed. Based on these results, further investigations were made to determine the shrinking and swelling kinetics by using the logistic curve equation and the Solver plug-in for Microsoft Excel (Eq. 2). In Fig. S1 and S2 (ESI) detailed views of the flow rate and the modelled flow rate can be seen.

\section{Results and Discussion}

\subsection{Temperature response of the hydrogel disks}

In the case of conventional temperature-responsive linear polymers, such as poly $(\mathrm{N}$ isopropylacrylamide), the LCST appears as a sharp transition at a specific temperature value $\left(\sim 32{ }^{\circ} \mathrm{C} \text { for pNiPAAm }\right)^{34}$. This remains valid also for linear PILs. In contrast, with crosslinked PILs the polymeric volume changes occur over a temperature interval, rather than at a sharp value, suggesting that more gradual changes are occurring in the polymer chains ${ }^{31}$. 
This phenomenon has been assigned to the decreased level of freedom of the bulky IL in the polymer network and manifests itself in an incremental decrease in size of the PIL hydrogels with increase in temperature (and vice versa). This behaviour also appears to occur in the PSPA hydrogels presented in this study.

Figure 4 shows that when the temperature is increased from $20{ }^{\circ} \mathrm{C}$ to $70{ }^{\circ} \mathrm{C}$ the hydrogels shrink by $\sim 39 \%$ at $50{ }^{\circ} \mathrm{C}$ and $\sim 56 \%$ at $70{ }^{\circ} \mathrm{C}$ (Video S1). When the temperature is lowered back from $70{ }^{\circ} \mathrm{C}$ to $20^{\circ} \mathrm{C}$, the hydrogels start absorbing water until they return to their original size. The slight difference in area between the heating and the cooling cycle arrose from the fact that the heating rate is $\sim 10{ }^{\circ} \mathrm{C} \cdot \mathrm{min}^{-1}$, while the cooling rate is $\sim 13{ }^{\circ} \mathrm{C} \cdot \mathrm{min}^{-1}$.

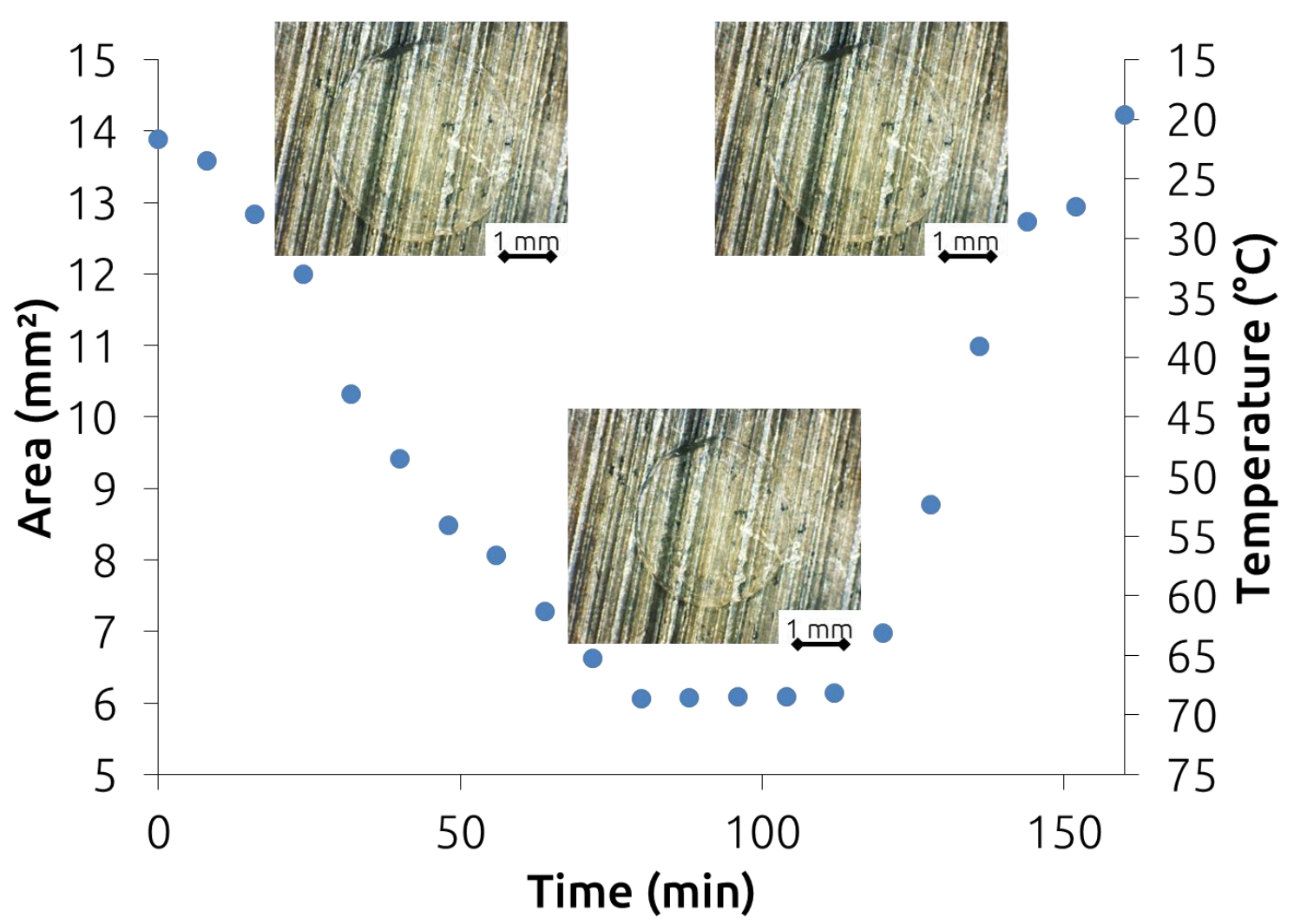

Fig. 4. The shrinking and reswelling behaviour of the PSPA hydrogels when the temperature is increased from $20{ }^{\circ} \mathrm{C}$ to $70{ }^{\circ} \mathrm{C}$, followed by decreasing it to $20{ }^{\circ} \mathrm{C}$. 
Figure 5 presents results of reproducibility and repeatability of this behaviour. In these experiments, a set of three gels were analysed by heating their hydration medium to a temperature of $50{ }^{\circ} \mathrm{C}$ to make the hydrogels shrink, followed by cooling them to $20{ }^{\circ} \mathrm{C}$ to allow for reswelling to their original size. As in the previous test, the hydrogels were kept for 8 min at each temperature, to ensure a steady-state volume was reached. The heating and cooling cycle was repeated six times. In all cases the hydrogels shrank by $\sim 44 \%( \pm 3 \%, \mathrm{n}=$ 6) at $50{ }^{\circ} \mathrm{C}$ and reverted back to $\sim 97 \%( \pm 2 \%, \mathrm{n}=6)$ of their original size when the temperature was lowered to $20^{\circ} \mathrm{C}$.

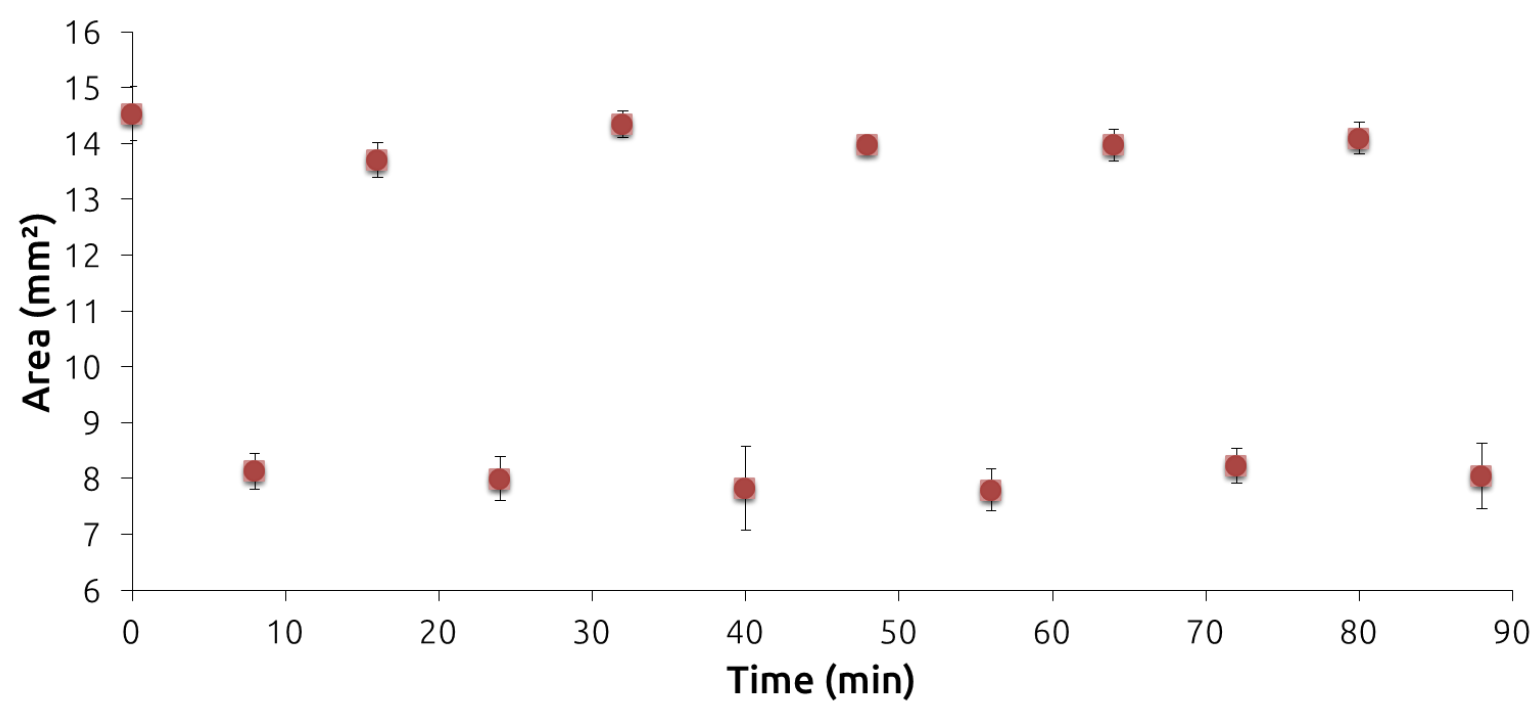

Fig. 5. Temperature-induced shrinking by area of the PSPA hydrogels at $20{ }^{\circ} \mathrm{C}$ and at $50{ }^{\circ} \mathrm{C}$.

\subsection{Flow characterization study}

Following this, microfluidic devices were fabricated as described in the experimental section with the necessary configuration for a hydrogel to be photopolymerised and used as a temperature-controlled valve. The valve was opened and closed six times (Fig. 6), thus confirming the reproducibility and repeatability of the valve thermo-actuation. 


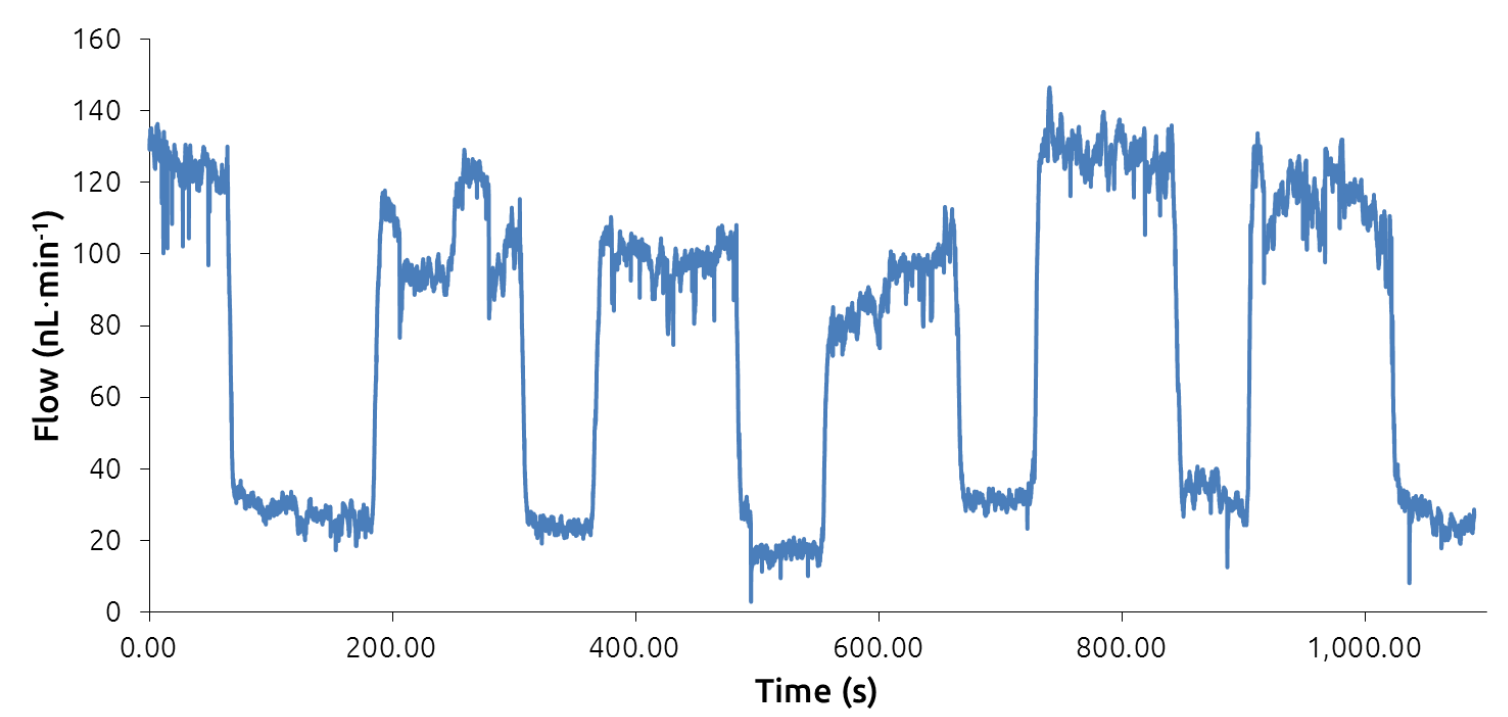

Fig. 6. PSPA hydrogel valve open at $50{ }^{\circ} \mathrm{C}$ with a flow rate of $\sim 140 \mathrm{~nL} \cdot \mathrm{min}^{-1}$ and closed at $20{ }^{\circ} \mathrm{C}$ with a flow rate of $\sim 20 \mathrm{~nL} \cdot \mathrm{min}^{-1}$. Small fluctuations on the signal due to ambient conditions (e.g. vibration of the pumping system) and the ionic liquid polymer network internal reorganisation before and after temperature actuation were observed $\left(10 \pm 5 \mathrm{~nL} \cdot \mathrm{min}^{-}\right.$ $\left.{ }^{1}\right)$.

It can be observed by the results that when valve is "closed" the flow sensor is able to detect a residual flow of $20 \mathrm{~nL} \mathrm{~min}{ }^{-1}$ instead of being 0 . The reason why the flow signal does not reach $0 \mathrm{~nL} \min ^{-1}$ can be attributed to:

(1) an inadequate assembly of the ionic liquid polymer microvalve within the microchannel walls. In order to minimise this effect, other configurations could be fabricated to better accommodate the generated microvalve.

(2) the polymer porosity; which could be reduced by increasing the concentration of crosslinker, but this will have an effect on the actuation times.

Similar behaviours have been obtained by us with other type of valves integrated in microfluidic devices ${ }^{17}$. 
In the other hand, a flow of $120 \mathrm{~nL} \mathrm{~min}{ }^{-1}$, when polymer is fully actuated, insted of $500 \mathrm{~nL}$ $\min ^{-1}$ means that the ionic liquid polymer is partially covering the microchannel, acting as a half open pipe. This is very common with this type of devices since it is very difficult to control the final swollen dimensions of the valve and so accordantly define the dimensions of the channel and reservoir.

The shrinking and swelling rate constants of the hydrogel valves (Table 1) were calculated using the logistic curve equation (Eq. 2) based on the flow rate measurements (Fig. 6).

Table 1. Experimental shrinking and swelling rate constants.

\begin{tabular}{|c|c|c|c|}
\hline Shrinking & & Swelling & \\
\hline Repetition & $\mathrm{k}$ & Repetition & $\mathrm{k}$ \\
\hline 1 & 0,9 & 1 & 0,9 \\
\hline 2 & 0,4 & 2 & 0,9 \\
\hline 3 & 0,5 & 3 & 0,8 \\
\hline 4 & 0,8 & 4 & 0,4 \\
\hline 5 & 0,7 & 5 & 0,5 \\
\hline 6 & 0,6 & & 0,7 \\
\hline Average & 0,6 & Average & 0,3 \\
\hline Standard Deviation & 0,2 & Standard Deviation & 36,4 \\
\hline RSD (\%) & 29,8 & RSD (\%) & \\
\hline
\end{tabular}

The rate constants were found to be $(1.1 \pm 0.3) \cdot 10^{-2} \mathrm{~s}^{-1}(\mathrm{n}=6)$ for the shrinking of the hydrogel and $(1.1 \pm 0.5) \cdot 10^{-2} \mathrm{~s}^{-1}(\mathrm{n}=5)$ for the swelling of the hydrogel. The close values of the rate constants convey the fact that both effects take place with approximately the same speed, which indicates no perceptible hysteresis between the opening and the closing of the PILc hydrogel valve when the temperature was cycled between $50^{\circ} \mathrm{C}$ and $20^{\circ} \mathrm{C}$ (SI, Fig. S1 
and Fig. S2). This effect is confirmed by the fact that the closing and opening time of the hydrogel valves vary between 5 to $8 \mathrm{~s}$. The actuation time values compare favourably with actuation times given for other stimuli-responsive hydrogels valves based on conventional thermo-responsive polymers ${ }^{52}$, or several phase-change mechanical actuators based on paraffin wax ${ }^{6}$, while being comparable with state of the art in hydrogel valves ${ }^{37,53}$.

\section{Conclusion}

In conclusion, a tributylhexyl phosphonium sulfopropyl acrylate ionic liquid monomer was synthesised and photopolymerised to produce a poly(ionic liquid) hydrogel that features a lower critical solution temperature. Due of this, the hydrogel is able to shrink by increasing the temperature of its surrounding hydration medium. Based on this thermoresponsive behaviour, the hydrogel actuation behaviour was fully characterised and the hydrogel was incorporated into a microfluidic device with the purpose of being used as temperature-controlled valve. The characterisation of the hydrogel indicates that the hydrogel shrinks by $\sim 58 \%$ of its swollen area when the temperature is raised from 20 to $70{ }^{\circ} \mathrm{C}$ and $~$ $39 \%$ when the temperature is raised from 20 to $50{ }^{\circ} \mathrm{C}$. Furthermore, the hydrogel is able to shrink and reswell repeatedly when subjected to temperature cycles between $20{ }^{\circ} \mathrm{C}$ and $50{ }^{\circ} \mathrm{C}$. Moreover, by including the hydrogel in a microfluidic device as a temperaturecontrolled valve, the microfluidic channel was modulated between $\sim 110 \mathrm{~nL} \cdot \min ^{-1}( \pm 15$ $\left.\mathrm{nL} \cdot \min ^{-1}, \mathrm{n}=6\right)$ and $\sim 27 \mathrm{~nL} \cdot \min ^{-1}\left( \pm 5 \mathrm{~nL} \cdot \min ^{-1}, \mathrm{n}=6\right)$ by varying the temperature between $50{ }^{\circ} \mathrm{C}$ and $20^{\circ} \mathrm{C}$. This process was repeated six times with no performance loss, thus suggesting the possibility of repeatable use for more than six times. Based on these results and taking into account the vast collection of ionic liquids available and their synthetic versatility, thermo-responsive poly(ionic liquid)s could constitute a new tool box for the generation of simple and low-cost temperature responsive microvalves. 


\section{Acknowledgements}

A.T., L.F., and D.D. are grateful for financial support from the Marie Curie Initial Training Network funded by the European Community's FP7 People Programme OrgBIO (Marie Curie ITN, GA607896) and Science Foundation Ireland (SFI) under the Insight Centre for Data Analytics initiative, Grant Number SFI/12/RC/2289. F.B.L. acknowledges the Ramón y Cajal Programme (Ministerio de Economía y Competitividad) and to Marian M. De Pancorbo for letting him to use her laboratory facilities at UPV/EHU.

\section{References}

1. P. N. Nge, C. I. Rogers and A. T. Woolley, Chemical Reviews, 2013, 113, 2550-2583.

2. G. M. Whitesides, Nature, 2006, 442, 368-373.

3. L. Dong and H. Jiang, Soft Matter, 2007, 3, 1223.

4. I. Araci and P. Brisk, Current Opinion in Biotechnology, 2014, 25, 60-68.

5. M. I. Mohammed, E. Abraham and M. P. Y. Desmulliez, Journal of Micromechanics and Microengineering, 2013, 23, 35034.

6. S. Ogden, L. Klintberg, G. Thornell, K. Hjort and R. Bodén, Microfluidics and Nanofluidics, 2014, 17, 53-71.

7. K. W. Oh and C. H. Ahn, Journal of Micromechanics and Microengineering, 2006, 16.

8. P. Selvaganapathy, E. T. Carlen and C. H. Mastrangelo, Sensors and Actuators A: Physical, 2003, 104, 275-282.

9. B. Yang and Q. Lin, Sensors and Actuators A: Physical, 2007, 134, 194-200.

10. C. Zhang, D. Xing and Y. Li, Biotechnology Advances, 2007, 25, 483-514.

11. D. J. Beebe, J. S. Moore, J. M. Bauer, Q. Yu, R. H. Liu, C. Devadoss and B.-H. Jo, Nature, 2000, 404, 588-590.

12. A. K. Agarwal, L. Dong, D. J. Beebe and H. Jiang, Lab on a Chip, 2007, 7, 310-315.

13. L. Florea, D. Diamond and F. Benito-Lopez, Research Perspectives on Functional Micro-and Nanoscale Coatings, 2016, 265.

14. J. ter Schiphorst, S. Coleman, J. E. Stumpel, A. Ben Azouz, D. Diamond and A. P. Schenning, Chemistry of Materials, 2015, 27, 5925-5931.

15. B. Ziółkowski, M. Czugala and D. Diamond, Journal of Intelligent Material Systems and Structures, 2012, 1045389-12459591.

16. M. Czugala, C. O’Connell, C. Blin, P. Fischer, K. J. Fraser, F. Benito-Lopez and D. Diamond, Sensors and Actuators B: Chemical, 2014, 194, 105-113.

17. J. Saez, J. Etxebarria, M. Antoñana-Diez and F. Benito-Lopez, Sensors and Actuators B: Chemical, 2016, 234, 1-7.

18. Y. Men, X.-H. Li, M. Antonietti and J. Yuan, Polymer Chemistry, 2012, 3, 871.

19. R. Bogue, Assembly Automation, 2012, 32, 37.

20. C. Boutris, E. G. Chatzi and C. Kiparissides, Polymer, 1997, 38, 2567-2570.

21. L. Florea, D. Diamond and F. Benito - Lopez, Macromolecular Materials and Engineering, 2012, 297, 11481159.

22. S. Gallagher, L. Florea, K. J. Fraser and D. Diamond, International Journal of Molecular Sciences, 2014, 15, 5337-5349.

23. S. Gallagher, A. Kavanagh, B. Zíołkowski, L. Florea, D. R. MacFarlane, K. Fraser and D. Diamond, Physical Chemistry Chemical Physics, 2013, 16, 3610.

24. M. R. Guilherme, R. da Silva, A. F. Rubira, G. Geuskens and E. C. Muniz, Reactive \& Functional Polymers, 2004, 61, 233-243. 
25. M. Hammarson, J. R. Nilsson, S. Li, T. Beke-Somfai and J. Andréasson, The Journal of Physical Chemistry B, 2013, 117, 13561-13571.

26. L. Ionov, Materials Today, 2014, 17, 494-503.

27. Y. Kohno, Y. Deguchi and H. Ohno, Chemical Communications, 2012, 48, 1188311885.

28. Y. Kohno and H. Ohno, Australian Journal of Chemistry, 2012, 65, 91-94.

29. Y. Kohno, S. Saita, Y. Men, J. Yuan and H. Ohno, Polymer Chemistry, 2015, 21632178.

30. J. Yuan, D. Mecerreyes and M. Antonietti, Progress in Polymer Science, 2013, 38, 1009-1036.

31. B. Ziółkowski and D. Diamond, Chemical Communications, 2013, 49, 10308-10310.

32. B. Ziółkowski, L. Florea, J. Theobald, F. Benito-Lopez and D. Diamond, Soft Matter, 2013, 9, 8754.

33. Y. Maeda, T. Higuchi and I. Ikeda, Langmuir, 2001, 17, 7535-7539.

34. H. G. Schild, Progress in Polymer Science, 1992, 17, 163-249.

35. X. Z. Zhang, Y. Y. Yang, T. S. Chung and K. X. Ma, Langmuir, 2001, 17, 6094-6099.

36. T. Glennon, J. Saez, M. Czugala, L. Florea, E. McNamara, K. J. Fraser, J. Ducree, D. Diamond and F. Benito-Lopez, ieee, 2015, 109-112.

37. F. Benito-Lopez, R. Byrne, A. M. Răduţă, N. E. Vrana, G. McGuinness and D. Diamond, Lab on a Chip, 2010, 10, 195-201.

38. S. Sugiura, K. Sumaru, K. Ohi, K. Hiroki, T. Takagi and T. Kanamori, Sensors and Actuators A: Physical, 2007, 140, 176-184.

39. N. S. Satarkar, W. Zhang, R. E. Eitel and J. Z. Hilt, Lab on a Chip, 2009, 9, 17731779.

40. S. Ghosh, C. Yang, T. Cai, Z. Hu and A. Neogi, Journal of Physics D: Applied Physics, 2009, 42, 135501.

41. G. Vancoillie, D. Frank and R. Hoogenboom, Progress in Polymer Science, 2014, 39, 1074-1095.

42. D. Mecerreyes, Progress in Polymer Science, 2011, 36, 1629-1648.

43. F. Benito-Lopez, M. Antoñana-Díez, V. F. Curto, D. Diamond and V. Castro-López, Lab on a Chip, 2014, 14, 3530-3538.

44. M. Yoshizawa and H. Ohno, Electrochimica Acta, 2001, 46, 1723-1728.

45. A. S. Shaplov, E. I. Lozinskaya, D. O. Ponkratov, I. A. Malyshkina, F. Vidal, P.-H. Aubert, O. g. V. Okatova, G. M. Pavlov, L. I. Komarova, C. Wandrey and Y. S. Vygodskii, Electrochimica Acta, 2011, 57, 74-90.

46. M. Koebe, M. Drechsler, J. Weber and J. Yuan, Macromolecular Rapid Communications, 2012, 33, 646-651.

47. S. Gallagher, L. Florea, K. Fraser and D. Diamond, International Journal of Molecular Sciences, 2014, 15, 53375349.

48. A. Tudor, L. Florea, S. Gallagher, J. Burns and D. Diamond, Sensors, 2016, 16, 219.

49. B. Ziółkowski, Z. Ates, S. Gallagher, R. Byrne, A. Heise, K. J. Fraser and D. Diamond, Macromolecular Chemistry and Physics, 2013, 214, 787796.

50. Y. Men, H. Schlaad, A. Voelkel and J. Yuan, Polymer Chemistry, 2014, 5, 37193724.

51. Y. Tanaka, T. Fujikawa, Y. Kazoe and T. Kitamori, Sensors and Actuators B: Chemical, 2013, 184, 163-169.

52. C. H. Zhu, Y. Lu, J. Peng, J. F. Chen and S. H. Yu, Advanced Functional Materials, 2012, 22, 4017-4022.

53. E. Lee, H. Lee, S. I. Yoo and J. Yoon, ACS applied materials \& interfaces, 2014, 6, 16949-16955. 


\section{Vitae}

Alexandru Tudor is a $\mathrm{PhD}$ student in Prof. Dermot Diamond's research group at Dublin City University. He graduated from the University "Politehnica" of Timisoara, Romania with a M. Sc. in Micro and Nanomaterials, and the West University of Timisoara, Romania with a B. Sc. in Chemistry. Alexandru's PhD programme is part of the OrgBIO FP7 project. His research focuses on the synthesis and characterization of poly(ionic liquid)s for use as smart polymers and functional materials in organic bioelectronics.

Janire Saez has a M.Sc. in chemistry (2012) and a postgraduate master in Pharmacology (2013) from the University of the Basque Country UPV/EHU in Spain. In 2015 she joined the Microfluidics Cluster UPV/EHU where is currently doing her $\mathrm{PhD}$ studies. Her research interests are focused on the integration of smart materials into environmentally friendly microfluidic devices.

Dr. Larisa Florea studied organic chemistry and chemical engineering at University "Politehnica" from Timisoara, Romania (B.Sc. Hons 2009). In 2009 she joined the Adaptive Sensors Group at Dublin City University where she earned her Ph.D. under the supervision of Prof. Dermot Diamond and Dr. Fernando Benito-Lopez. Since 2013 she has carried out her postdoctoral research with Prof. Dermot Diamond in the INSIGHT Centre at Dublin City University, where she is currently Team Leader in smart materials and microfluidics. Her research interests include the design, synthesis and applications of stimuli-responsive polymers as novel sensing and actuating materials in microfluidic devices.

Dr. Fernando Benito-Lopez studied chemistry at the Universidad Autonoma de Madrid and completed his master studies in the Department of Inorganic Chemistry in 2002.He obtained his $\mathrm{PhD}$ at the University of Twente, The Netherlands, in 2007. He carried out his postdoctoral research in the group of Prof. Dermot Diamond at Dublin City University, Dublin, where in 2010, he became Team Leader in polymer microfluidics. In 2012 he moved to CIC microGUNE a Research Centre working in Microtechnology in Spain. From 2015 he is Ramón y Cajal Fellow and leader of the Microfluidics Cluster UPV/EHU at the University of the Basque Country, Spain. 
Prof. Dermot Diamond received his Ph.D. and D.Sc. from Queen's University Belfast (Chemical Sensors, 1987, Internet Scale Sensing, 2002), and was Vice-President for Research at Dublin City University (2002-2004). He has published over 300 peer-reviewed papers in international journals, is a named inventor in 18 patents, and is co-author and editor of four books. He is director and founding member of the National Centre for Sensor Research (www.ncsr.ie) at Dublin City University, and an SFI-funded investigator in the INSIGHT Centre (http://www.insight-centre.org). 